\title{
Impact of dietary nutrients (functional foods/nutraceuticals) and mi- cronutrients on COVID-19: a review
}

\author{
Yueching Wong ${ }^{\mathrm{a}}$, Chi-Ho Chan ${ }^{\mathrm{b}}$, Kamesh Venkatakrishnan ${ }^{\mathrm{a}}$, Hui-Fang Chiuc ${ }^{\mathrm{c}}$, You-Cheng Shen ${ }^{\mathrm{d}}$, \\ Oksana Glovinskaia ${ }^{\mathrm{a}}$, Yi-Chun Han ${ }^{\mathrm{e}}$ and Chin-Kun Wang ${ }^{\mathrm{a}^{*}}$
}

\begin{abstract}
aDepartment of Nutrition, Chung Shan Medical University, 110, Sec. 1, Jianguo North Road, Taichung City, Taiwan, ROC
${ }^{b}$ Department of Medical Research, Chung Shan Medical University Hospital, Department of Microbiology and Immunology, Chung Shan Medical University, 110, Sec. 1, Jianguo North Road, Taichung City, Taiwan, ROC

'Department of Chinese Medicine, Taichung Hospital Ministry of Health and Well-being, Taichung, Taiwan, ROC

${ }^{\mathrm{d} D e p a r t m e n t ~ o f ~ H e a l t h ~ I n d u s t r y ~ T e c h n o l o g y ~ M a n a g e m e n t, ~ C h u n g ~ S h a n ~ M e d i c a l ~ U n i v e r s i t y, ~ 110, ~ S e c . ~ 1, ~ J i a n g u o ~ N o r t h ~ R o a d, ~ T a i c h u n g ~}$

City, Taiwan, ROC

eDepartment of nutrition and health science, Fooyin University, Kaohsiung, Taiwan, ROC

*Corresponding author: Chin-Kun Wang, Department of Nutrition, Chung Shan Medical University, 110, Sec. 1, Jianguo North Road, Taichung City, Taiwan, ROC. Tel: +886 4 22653397; Fax:+886 4 22654529; E-mail: wck@csmu.edu.tw
\end{abstract}

DOI: $10.31665 /$ JFB. 2021.15280

Received: September 06, 2021; Revised received \& accepted: September 29, 2021

Citation: Wong, Y., Chan, C.-H., Venkatakrishnan, K., Chiu, H.-F., Shen, Y.-C., Glovinskaia, O., Han, Y.-C., and Wang, C.-K. (2021). Impact of dietary nutrients (functional foods/nutraceuticals) and micronutrients on COVID-19: a review. J. Food Bioact. 15: 29-38.

\begin{abstract}
The world health organization (WHO) announced that coronavirus 2019 (COVID-19) is a pandemic. Considering the pandemic spread of Covid-19, many researchers have shown immense interest in various functional foods/ nutraceuticals and dietary supplements to improve immune function and overall health status and thus lower the risk of COVID-19 infection. Studies have indicated that a balanced diet rich in various nutrients especially micronutrients plays a huge role in recovering and preventing COVID-19 related health issues. Improvement of obesity could reduce the risk of COVID-19 infection owing to the less angiotensin-conversion enzyme 2 receptors and increase the effect of vaccination. This review signifies the importance of various food bioactives along with dietary supplements (balanced diet) rich in various micronutrients against COVID-19 and its related anomalies. Moreover, this contribution helps non-specialists to understand the importance of various functional foods/nutraceuticals based on the dietary/supplementation recommendations indicated by various popular nutritional or dietary or health organizations. To sum up this contribution, healthy life style and balanced nutrition play important role on immune system. Reducing obesity becomes a critical point on the COVID-19 infection. The most important is to find the potential and effective food bioactives, which might act as supportive or complementary therapy (prophylactic) for mitigating the risk and comorbidity associated with COVID-19.
\end{abstract}

Keywords: Coronavirus 2019 (COVID-19); Functional foods; Nutraceuticals; Dietary supplements; Micronutrients.

\section{Introduction}

Severe acute respiratory syndrome coronavirus 2 (SARS-CoV2) or coronavirus causes a pneumonia-like respiratory disease, which is commonly called COVID-19 (Coronavirus-2019). Coronavirus is a positive sense single standard RNA (ssRNA), which is enveloped by a spike glycoprotein, which appears like a crown under a 2D-electron microscope, and hence it is generally called a coronavirus (Iddir et al., 2020). Coronavirus is a member of the coronaviridae family and has a close connection with severe acute respiratory syndrome (SARS) and Middle East respiratory syndrome (MERS). Coronavirus structure consists of four major 
proteins including spike glycoprotein, nucleoprotein (attached with RNA), membrane protein, and an envelope protein, which plays a key role in replication (division). SARS-CoV2 can use angiotensin-converting enzyme 2 (ACE2) as a receptor and enter host cells and start to divide and finally cause COVID-19 (Mrityunjaya et al., 2020; Wiersinga et al., 2020). Concomitant Obesity and related health issues become the targets of COVID-19 infection and indicator of severe syndrome, and the efficacy of vaccination is suppressed by obesity (Goossens et al., 2020; Bakhshandeh et al., 2021). The novel coronavirus (SARS-CoV2) outbreak from Wuhan, China has inflicted a global emergency health threat to humanity since December 2019. This coronavirus pandemic has impacted the economy and health care system all over the world (Kozlov et al., 2021). This coronavirus spreads from human to human via respiratory and airborne droplets as well as through direct contacts. The biggest threat of the coronavirus is that most of the infected patients are asymptomatic and few develop severe symptoms like fever, dry cough, fatigue loss of smell/taste, and headache. If COVID-19 is not treated properly it can result in high mortality and morbidity and also its long-term effects are not clear (Kazemi et al., 2021; Syed, 2020). Especially, elderly people, people with comorbid conditions (Cardiovascular, diabetes mellitus, cancer, and hypertension), immunosuppressed subjects, and heavy smokers are highly susceptible to Coronavirus infection (Subedi et al., 2021). Recently, WHO has announced four major variants/ genera of COVID-19 virus including $\alpha, \beta, \gamma, \delta$ till date (out of 11 known variants). Out of all these coronavirus variants the $\delta$ (delta) is one the deadliest with a high transmissibility rate (Reproductive number) and contributed to high mortality and morbidity globally in recent times (Alizon et al., 2021; Liu et al., 2021).

\section{An overview on the pathophysiology of COVID-19}

The detailed pathophysiology of COVID-19 is still being explored. However, few major risk factors like oxidative stress, inflammation, and altered immune response are assumed to be the major contributor to COVID-19 and its related health issues (Kozlov et al., 2021; Iddir et al., 2020). In this section, we would like to discuss the connection between oxidative stress, inflammation, and immune response and how they contribute to COVID-19. Once the SARS-CoV2 enters host cells (mostly lung cells) via ACE2 receptor by endocytosis, it starts to replicate and affects the cellular homeostasis, resulting in oxidative stress (increased free radical production and reduced antioxidant activity), which inflicts an imbalance between free radicals and antioxidants. These excessive free radicals (reactive oxygen species [ROS]/reactive nitrogen species [RNS]) could alter various signaling pathways that are responsible for various host cellular metabolic processes (Hussain et al., 2016). These excessive ROS/RNS triggers inflammatory response via toll-like receptor (TLR) and nuclear factor-kappa B (NF-kB) signaling pathway by increasing the production of various proinflammatory cytokines. The excessive production of inflammatory cytokines results in hyperinflammatory condition (cytokines storm) and also alters the release and activation of various immune cells especially monocytes, neutrophils, and macrophages (Wiersinga et al., 2020; Leisman et al., 2020). These activated immune cells further increase the production of free radicals by suppressing the nuclear factor erythroid related factor 2 (Nrf2) signaling pathway, which is responsible for endogenous antioxidant (cytoprotection) and detoxification related enzymes (Laforge et al., 2020). In addition, elevated cytokines trigger the induction of endothelium HA-Synthase-2 (HAS2) and subsequently increase the production of hyaluronan (HA), which has a high water binding capacity and eventually results in increased accumulation of lung fluid (sepsis). As mentioned before SARS-CoV2 mostly enters the host system through the respiratory tract and hence lungs cells are highly affected by oxidative stress, inflammation, and altered immune response and result in fibrosis, fluid accumulation, and sepsis (Shi et al., 2020). Hence, most of COVID-19 patients have various lung or respiratory-related problems especially acute respiratory distress syndrome (ARDS), and pneumonia (Kellner et al., 2017). Both oxidative stress and inflammation are strongly interconnected in that one activates another (vicious cycle). Similarly, oxidative stress and inflammation result in altered immune response (innate and acquired immunity) which plays a dominant role in the pathophysiology of COVID-19 (Leisman et al., 2020). Furthermore, studies have indicated that elevated ROS production (oxidative burs/ stress), cytokine storm (inflammation), immune dysfunction end up in multi-organ failure by altering various normal cellular functions like autophagy, apoptosis, necrosis (cell death), cell energy system (mitochondrial dysfunction) along with altered microbiota flora (microbiota dysbiosis) could increase the severity of SARSCoV2 infection (Yuki et al., 2020; Smith et al., 2020). Because, of the above-mentioned mechanism (pathophysiology), COVID-19 patients start to develop various comorbid conditions which eventually leads to higher mortality and morbidity. Those individuals who already have comorbid conditions like hypertension, diabetes mellitus, cardiovascular or renal/lung-related disorders are highly vulnerable to high morbidity and mortality (Liu et al., 2020).

\section{Modified lifestyle pattern to lower the risk of COVID-19}

So far, we have given an overview of COVID-19 and its impacts on humans by explaining various pathophysiological events and their associated factors. We would now like to emphasize the importance of a modified lifestyle pattern for reducing the risk of COVID-19 as well as some recommendations for a speedy recovery from COVID-19. The common recommendation from various expert includes regular exercise (indoor or outdoor), maintaining social distancing, wearing a mask and washing hands (hygienic lifestyle) as well as limiting smoking and alcohol consumption (Ismail et al., 2021; WHO, 2020). In addition, consumption of healthy foods including regular intake of fruits, vegetables, dietary fibers, nuts, and cereals along with various dietary supplements would lower the risk of COVID-19. Even though various COVID-19 vaccines are available in the market, but it does not provide $100 \%$ protection against COVID-19, as well as its long-term adverse effects are largely unknown (Subedi et al., 2021; Cascella et al., 2021). Moreover, the vaccine can only help to improve or boost the immune response against coronavirus (produce antibodies). However, the individual immune system should be strong enough to produce enough antibodies (plasma/memory cells) to fight against the coronavirus (Ahn et al., 2020). Hence, consumption of a healthy balanced diet would help to boost the host immune system and thereby mitigate the harmful health impacts associated with coronavirus. Along with vaccination, few anti-viral drugs and micronutrients are recommended for treating Covid-19 but following a healthy lifestyle (healthy food) would be the best choice to help combating this pandemic (Lordan et al., 2021).

One very important issue is obesity control. Obesity and related health issues (hypertension, diabetes and coronary heart diseases) are well known as main reasons of infection and severe syndrome of COVID-19 and vaccination efficacy owing to rich ACE2 receptor (Goossens et al., 2020; Bakhshandeh et al., 2021). ACE2 receptor 
Table 1. Summary of dietary/supplementation recommendations and proposed health benefits along with food hygienic guidelines from different nutritional or dietary or health organizations/societies/councils

\begin{tabular}{|c|c|c|}
\hline Dietary/supplementation recommendation & $\begin{array}{l}\text { Proposed health benefits and hy- } \\
\text { gienic guidelines (food safety) }\end{array}$ & $\begin{array}{l}\text { Organization/As- } \\
\text { sociation/Council }\end{array}$ \\
\hline $\begin{array}{l}\text { Intake of fresh fruits and vegetables rich in } \\
\text { fibers, minerals, and vitamins (Colored fruits } \\
\text { and vegetables are highly recommended). } \\
\text { Avoid or limit the use of processed or canned } \\
\text { food. Lower the consumption of sugar, fat, or } \\
\text { salt. Keep hydrated (drink enough water). Do } \\
\text { some indoor exercises, yoga, and meditation }\end{array}$ & $\begin{array}{l}\text { Consumption of fruits and vegetables can improve overall } \\
\text { health status especially lower oxidative stress and enhance } \\
\text { immune system and thus lowering the risk of Covid } \\
\text { 19. Wash hands before handling food (before eating or } \\
\text { cooking). Wash vegetables/fruits before cooking. Maintain } \\
\text { social distancing and wear a mask (outdoor activities) }\end{array}$ & WHO (2020) \\
\hline $\begin{array}{l}\text { Increase fruits and vegetable consumption. Intake } \\
\text { fish oil rich in DHA/EPA. Vitamin and mineral- } \\
\text { rich supplements or fruits/vegetables must be } \\
\text { consumed. Drink a glass of milk and take only } \\
\text { low-fat dairy products are better (especially } \\
\text { probiotics). Lower the usage of processed } \\
\text { and junk foods. Make cooking and eating fun, } \\
\text { makes children happy, and lower stress }\end{array}$ & $\begin{array}{l}\text { Consumption of fruits and vegetables rich in vitamins } \\
\text { and minerals could decrease stress and enhance the } \\
\text { endurance and immune response (increase antibody } \\
\text { production) and thus mitigate the severity of Coronavirus } \\
\text { infection. Wash hands before handling food (before } \\
\text { eating or cooking). Pregnant women with Covid } 19 \\
\text { can still breastfeed. Children should maintain social } \\
\text { distancing and wear a mask (outdoor activities) }\end{array}$ & UNICEF (2020) \\
\hline $\begin{array}{l}\text { Consume a healthy and balanced diet by } \\
\text { including vegetables, nuts (healthy fat), whole } \\
\text { grains, and fruits. Diversity food consumption } \\
\text { and do not avoid any food completely, to } \\
\text { balance your system. Limit alcohol consumption. } \\
\text { Avoid excessive intake of fats or sugar }\end{array}$ & $\begin{array}{l}\text { Eating healthy and balanced food will significantly improve } \\
\text { the immune system (proliferation of T and B cells). } \\
\text { Dietary fibers and healthy fat can lower the comorbid } \\
\text { conditions associated with Covid } 19 . \text { Wash all the food } \\
\text { materials before cooking. Wash hands regularly }\end{array}$ & FAO (2020) \\
\hline $\begin{array}{l}\text { Prefer fresh food and try to avoid stored or } \\
\text { canned food especially fruits and vegetables. } \\
\text { Consume dried nuts like walnut, almond, } \\
\text { cashews, peanuts. Intake whole grains/cereals } \\
\text { and drink at least a glass of milk. Try to consume } \\
\text { herbs and spices with your food. But avoid } \\
\text { excessive salt or sugar (carbonated drinks) }\end{array}$ & $\begin{array}{l}\text { Following the dietary plan, it can help maintaining a } \\
\text { strong immune function as well as substantially improve } \\
\text { your mental health. Sterilize the food before cooking } \\
\text { and wash hand with soap for at least } 20 \text { seconds }\end{array}$ & DAA (2020) \\
\hline $\begin{array}{l}\text { Recommended to control the disease by } \\
\text { following only hygienic procedure (food } \\
\text { safety), no dietary recommendation }\end{array}$ & $\begin{array}{l}\text { Wash your hand regularly for at least } 20 \text { seconds. } \\
\text { Clean/sanitize the working space and kitchen to } \\
\text { avoid food contamination. Refrigerate perishable } \\
\text { food and clean than before cooking. Wear a mask as } \\
\text { much as possible and maintain social distancing }\end{array}$ & CDC (2020) \\
\hline
\end{tabular}

is a protein on the surface of many cell types. It is an enzyme that generates small proteins, by cutting the larger protein angiotensinogen, that then go on to regulate functions in the cell. To prevent the infection and avoid severe syndrome of COVID-19 infection, concomitant obesity control is very critical and effective. Energy intake control and physical activity (healthy lifestyle) could be greatly encouraged at this time. Even though many strategies until now have been recommended to treat COVOD-19, but the way to help treating this disease is important. Food bioactives are treasures from nature which show great potential to treat COVID-19 problems. Basic studies together with clinical trials are required in the near future. The authors have set up a good model to screen a lot of potential and effective food bioactives on COVID-19 virus, some valuable and exciting results have so far been obtained. Our next step is clinical trial for getting the science-based evidence for them.

\section{Dietary guidelines from health/ dietary organization/soci- ety/council to prevent COVID-19}

Different nutritional or dietary or health organizations/societies/ councils, including World Health Organization (WHO), Food and Agriculture Organization (FAO) of the UN, and European Food Information Council (EFC), Center for Disease Control and Prevention (CDC), Dietary Association of Australia (DAA) and United National Children's Fund (UNICEF) have proposed various health/ dietary guidelines and advice pertaining to COVID-19 pandemic. Table 1 epitomizes the summary of health/dietary guidelines and advice indicated by various organizations or councils which mostly includes the consumption of fruits, vegetables, fish oil, probiotics, whole grains, cereals, and nuts. These recommended foods could substantially improve the immune system and thereby elicit overall protection from COVID-19 infection and lower the risk of Covid related health issues as well as improve the recovery rate in Covid infected subjects (WHO, 2020; UNICEF, 2020; FAO, 2020; DAA, 2020; de Faria Coelho-Ravagnani et al., 2021). Overall, all major health/ dietary organizations/societies/councils have recommended that individuals consume healthy food including fruits and vegetables (rich in fibers, minerals, and vitamins) along with drinking an adequate level of water (hydrated). In addition, avoiding smoking and reducing alcohol consumption have been suggested (de Faria Coelho-Ravagnani et al., 2021). 


\section{Importance of functional food/nutraceuticals and dietary supplements on COVID-19}

Considering this coronavirus pandemic outbreak, people are becoming highly health-conscious and have started to focus on healthy food. When it comes to healthy food, people mostly remember functional foods/nutraceuticals, herbs, and dietary supplements as they might be the better tool to combat against COVID-19 and its related health issues (Subedi et al., 2021). Both functional foods and nutraceuticals terms are often used interchangeably but there are subtle differences between them. In the case of functional food, they are considered as a traditional or conventional food or food byproduct, which could be fortified or altered to enhance its nutritional value and thus demonstrably enhance its overall biological function and health status (Venkatakrishnan et al., 2019; Shahidi, 2012). However, nutraceuticals are part of the food (bioactive) which can be used in the medicinal form or supplement to improve overall health status (Daliu et al., 2019). Recent studies have indicated that intake of functional foods/nutraceuticals and dietary supplements rich in various micronutrients would lower the risk of COVID-19 infection as well as speeding up the recovery in COVID-19 subjects since most viral infections can compromise immune function and lower micronutrient levels (especially vitamins/minerals). Moreover, functional foods/nutraceuticals and dietary supplements are well known for their antioxidant, anti-inflammatory, and immunomodulatory properties. Hence, they can effectively lower oxidative stress and inflammation and thereby positively regulate immune cells (immune booster) which eventually results in improving overall health status (Subedi et al., 2021; Lordan et al., 2021; Galanakis et al., 2020; Mrityunjaya et al., 2020). Both vitamins and minerals (micronutrients) play a central role in various cellular activities (metabolic function, immune function, antioxidant, anti-inflammation), structural/body development (growth), and thereby improve overall health status (Chiu et al., 2021). In this contribution, we havechosen only the popular nutraceuticals/functional food or micronutrients (dietary supplements) like probiotics, fish oil, and curcumin, and quercetin which have been reported to show potent anti-viral and anti-respiratory effects. Most of the clinical trials are still ongoing and only a few trials have come up with positive results, thus, it very hard to make any definite conclusions. Hence, we have referred to the previous data of various nutraceuticals/functional foods or micronutrients against viral infection which are related to respiratory disorders like lung sepsis, pneumonia, ARDS, SARS, and MERS, might be also effective against SARS-CoV2 or COVID-19 injection.

\section{Functional food/nutraceuticals against COVID-19}

\subsection{Probiotics (pre and post biotics)}

Probiotics are living microorganisms in the gut, which are believed to improve host health status, whereas, prebiotics are defined as non-digestible food acting as a food material that stimulates the growth of host beneficial gut microorganism (probiotics). Similarly, postbiotics are fermented by-products (of prebiotics) produced in the gut that help promoting health (Zolkiewicz et al., 2020). The popular probiotics species include Lactobacillus and Bifidobacterium spp., streptococcus (Beneficial bacteria's) which are commonly used in various commercial food and supplement products to enhance the composition of gut microbiota and hence used as functional foods/nutraceuticals (Chiu et al., 2021). Pro- and prebiotics also show various biological functions including anti-inflammatory, antioxidant, and immunomodulatory activity through modulating the gut-brain axis (Pandey et al., 2015). The proposed mechanism of pro- and prebiotics against COVID-19 include reduced expression of inflammatory markers by inhibiting TLR 2/4, NF- $\kappa \mathrm{B}$, and TNF- $\alpha$ signaling pathway, improving intestinal barrier function, and thus lowering the infiltration of inflammatory and immune cells (Akour, 2020). Various probiotic spp include Lactobacillus and Bifidobacterium which play a key role in clearing the influenza virus by inhibiting the attachment with the receptor (Baud et al., 2020). Likewise, previous studies also indicate that probiotics can inhibit the transmissible gastroenteritis virus from entering the epithelial cells and thus protect the upper respiratory and gastric tract from viral infection (Wang et al., 2017). Probiotics are reported to modulate immune function (innate immunity) by altering the production of NK and APCs cells (Chong et al., 2019; Mortaz et al., 2013). Clinical trials conducted by Zeng (2016) and Morrow et al. (2010) demonstrated that supplementation with prebiotics in respiratory ill subjects on ventilation showed less ventilator-associated pneumonia compared with the placebo group. In addition, several reports indicate that pre-/ pro- and postbiotics can be beneficial against COVID-19 infection and can lower the severity owing to their potent anti-inflammatory, antioxidant, anti-viral, and immune-modulatory properties via improving gut microbiota, which in turn enhance the gut-brain axis and gut-lung axis (Baud et al., 2020; Sundararaman et al., 2020; Akour, 2020). Based on the above data, probiotic/prebiotic supplementation may be used as prophylactic and supportive therapeutic property (used along with standard drugs) against COVID-19. However, more trials are needed before confirming their definite effect against the coronavirus.

\subsection{Fish oil (DHA/EPA)}

Fish oil is rich in various polyunsaturated fatty acids (PUFA) which are comprised of fatty acids with multiple double bonds. These include alpha-linolenic acid (ALA), docosahexaenoic acid (DHA) and eicosapentaenoic acid (EPA) which are classified as Omega $(\omega)$-3-fatty acids with an array of pharmaceutical properties including antioxidant and anti-inflammatory activities (Venkatakrishnan et al., 2019). The proposed mechanism of fish oil against COVID-19, especially DHA and EPA, is by exerting potent anti-inflammatory activity via suppressing the production of pro-inflammatory cytokines and Cox-2, and hence can attenuate cytokines Strom process (Szabo et al., 2020). Fish oil also acts as an ACE2 inhibitor and hence acts as an anti-corona viral agent. Adaptive and innate immunity activity of fish oil was demonstrated by altering the production of NK and neutrophil immune cells (Calder, 2007). Fish oil rich in DHA and EPA showed anti-platelet and anti-thrombotic/anti-atherogenic activity and thus used to treat COVID-19 and its related complications (Rogero et al., 2020). A preliminary trial showed that blood samples from 100 COVID-19 patients with a higher DHA/EPA index had a lower risk of death due to Covid-19 (Asher et al., 2021). Even though fish oil was found to be beneficial against COVID-19, but the limited clinical trials make it difficult to recommend fish oil for treating COVID-19.

\subsection{Curcumin}

Curcumin is one of the key active ingredients of turmeric. Turmeric is commonly used in Asian cuisine especially in India as 
a flavoring/coloring agent with numerous biological functions, and hence it is considered a popular nutraceutical/functional food ingredient (Chiu et al., 2021). The proposed mechanism of curcumin against COVID-19 is by exerting antiviral effects against Zika virus, Influenza virus, Human papillomavirus (HPV), and Human immunodeficiency virus (HIV) through inhibiting viral attachment and replication (Chen et al., 2013). In addition, curcumin can suppress ACE2 binding and thus inhibit or control SARS-CoV virus entry (Zahedipour et al., 2020). The available data demonstrate that curcumin can also work effectively against SARS-CoV (coronavirus). Curcumin has been reported to lower antioxidant stress via enhancing antioxidant activity (scavenge free radicals/ anti-lipid peroxidative function) as well as suppressing the production of inflammatory cytokines via inhibiting NF- $\kappa B$, STAT, and COX-2 signaling pathways (Rocha et al., 2020). It also stimulates host interferon production and thus trigger innate immunity (Hamid and Thakur 2021).

\subsection{Quercetin}

Quercetin is a flavonoid (flavanol) and is usually present in vegetables and fruits. Quercetin is available in both aglycone and glycosidic form (rutin) and has various biological functions including antioxidant, anti-inflammatory, ant-viral, and immunomodulatory activities (Venkatakrishnan et al., 2019). The proposed action mechanism of quercetin against COVID-19 is displayed by its strong anti-inflammatory activity and by inhibiting the production of various pro-inflammatory cytokines and inflammatory markers like COX-2 and LOX (Bhasker et al., 2011). It improves antioxidant status by upregulating the Nrf2 signaling pathway (Sharma et al., 2020). Previous studies indicate that quercetin can suppress the replication of RNA viruses-like influenza and coronavirus. Quercetin is reported to inhibit SARS CoV2 (spike protein) attachment to ACE2 and thus interfering with the endocytosis or entry of SARS CoV2 into cells and thus act as an anti-SARS CoV2 agent (Smith and Smith, 2020). Moreover, a better anti-corona virus effect was found with quercetin, when combined with vitamin $\mathrm{C}$, thus demonstrating their immunomodulatory and anti-viral properties. Furthermore, supplementation of vitamin C with Quercetin for 7 days was shown to improve the recovery rate (reduce hospital stay) in COVID-19 patients (Colunga Biancatelli et al., 2020).

\section{Micronutrients against COVID-19}

\subsection{Vitamins}

Vitamins are essential micronutrients, which are classified as water-soluble (B, C) and lipid-soluble (A, D, E, K). All the vitamins are involved in various biological functions including growth and development. Especially vitamins A, C, D, and E play a critical role in maintaining the immune system along with their antioxidant, anti-inflammatory and anti-viral activities which make them a special weapon in combating various upper respiratory illnesses. Hence vitamins A, C, D, and E might also work effectively against SARS- CoV2 or coronavirus (Dehghani-Samani et al., 2020; Jovic et al., 2020).

\subsection{Vitamin C (ascorbic acid)}

Vitamin $\mathrm{C}$ is a water-soluble vitamin that aids to improve immune function and is also involved in the development of body tissue (collagen formation) and speeds up the wound healing process. In addition, vitamin $\mathrm{C}$ has potent antioxidant, anti-inflammatory, and anti-viral properties, which might improve overall health status and thus helps in the management of Covid and its related complications like sepsis and pneumonia (Carr and Rowe, 2020). The possible mechanism of vitamin C against COVID-19 includes modulation of the activation of various immune cells (improve immunity) and thereby regulating the production of various inflammatory markers (suppress cytokine storm) and decreasing the histamine levels (Calder, 2020). Vitamin C can increase the differentiation and proliferation of $\mathrm{B}$ and T Cells and thus aid in the production of antibodies against SARS- CoV2 (Abobaker et al., 2020). Vitamin $\mathrm{C}$ has also been reported to enhance various antioxidant activities via upregulating the Nrf2 signaling pathway (Cheng, 2020) and can reduce the severity of upper respiratory viral infections like ARDS (Hagel et al., 2013). A pilot trial conducted by Zhang et al. (2021) has hinted that administration of vitamin $\mathrm{c}$ for 7 days in 56 ill covid subjects resulted in a significant reduction of hospital stay and mortality. Moreover, supplementation of vitamin $\mathrm{C}$ with quercetin for 7 days improved the recovery rate (reduce hospital stay) in COVID-19 patients (Colunga Biancatelli et al., 2020). Overall, vitamin C, owing to its potent antioxidant, anti-inflammatory, anti-viral, and immune-boosting properties, might help treating COVID-19 patients, however, more evidence is needed before its recommendation for treating COVID-19.

\subsection{Vitamin D or 25-hydroxyvitamin D}

Vitamin D is a fat-soluble vitamin that plays an important role in several physiological processes, such as bone metabolism, calcium, and phosphorus absorption, and enhance cellular immunity. Vitamin D is also showing promising results against various upper respiratory disorders or syndrome like ARDS. A mounting number of studies demonstrate that deficiency of vitamin D (hypovitaminosis D) is strongly associated with an increase in the incidences of COVID-19 and its severity (Liu et al., 2021; Pereira et al., 2020; Grant et al., 2020). However, few studies have shown no association between vitamin D and Coronavirus (Jevalikar et al., 2021; Murai et al., 2021). The proposed mechanism against COVID-19 includes intake of vitamin D that could lower inflammatory cytokines production (cytokines storm) via downregulating the NF- $\mathrm{KB}$ signaling pathway. Vitamin D could effectively modulate immune function by altering the activation of various immune cellsthus regulating the production of cytokines as well as increasing the production of antibodies against coronavirus (Mrityunjaya et al., 2020; Barlow et al., 2011). In addition, vitamin D is reported to induce cathelicidin (LL-37) and related peptides/protein and thus promoting innate immunity and eventually lowering the replication of SARS-CoV2 and influenza virus (Kazemi et al., 2021; Sharifi et al., 2019). In addition, vitamin D is involved in the regulation of renin-angiotensin-aldosterone system (RAAS) and angiotensin-converting enzyme 2 (ACE2), which are responsible for attachment of SARS CoV2 and COVID-19 infection (Pal et al., 2021). Vitamin D could upregulate the expression Nrf2 signaling pathway and thereby facilitating the balanced mitochondrial function (Mrityunjaya et al., 2020). A meta-analysis conducted by Martineau et al., (2017) found that vitamin D administration reduces the risk of upper respiratory infection. Pal et al. (2021) indicated that vitamin D supplementation (safe) significantly improved various clinical outcomes like a lower hospital stay, mortality, adverse events, and speedy recovery. A systemic review and meta-analysis conducted by Kazemi et al (2021) also concluded that a significant 
relationship exists between vitamin $\mathrm{D}$ and Covid infection, severity, and mortality. Especially vitamin D deficiency (VDD) has a direct association with Coronavirus infection and mortality. Even though vitamin D showed a promising effect against SARS-CoV2, but the dose, mode of administration, duration, and combination with other drugs or nutrients remains largely unknown, and hence further studies are needed before making any recommendations related to Covid patients.

\subsection{Vitamin A}

Vitamin A exhibits potent anti-inflammatory and immunomodulatory activities via regulating the cellular and humoral immune response. The possible mechanism underlying vitamin A protection against COVID-19, includes elevated production of T cells, NK cells, and neutrophils (Tanumihardjo et al., 2016). Previously, few researchers indicated that supplementation of vitamin A could significantly reduce the onset of various viral diseases like HIV, HPV as well as decreasing the mortality and morbidity associated with various respiratory illnesses and viral infections (Hamid and Thakur 2021; Villamor and Fawzi, 2005).

\subsection{Vitamin $E$}

Vitamin E is a potent antioxidant and plays a crucial role in maintaining immune function especially for the elderly. Vitamin $\mathrm{E}$ is also involved in $\mathrm{T}$ and $\mathrm{B}$ cell proliferation and differentiation as well as improving NK cell and APCs activity and thus regulating immune response (Junaid et al., 2020; Lee and Han, 2018). Vitamin $\mathrm{E}$ is a strong anti-inflammatory agent as it suppresses the production of pro-inflammatory cytokines (Lewis et al., 2019). Hence, it may be used to treat Covid related hyperinflammatory conditions along with its antioxidant and immunomodulatory activities. The evidence of vitamin E against COVID-19 is limited, but it might be used with other essential micronutrients as supportive therapy to enhance immunity. Moreover, vitamin $\mathrm{B}$ complex $\left(\mathrm{B}_{6}, \mathrm{~B}_{9}, \mathrm{~B}_{12}\right)$ also helps to improve immune function by enhancing the production of NK cells (innate immunity), T and B cells proliferation to enhance the antibodies production against coronavirus. However, only limited studies have been conducted on this topic so far and hence the association of vitamin B complex with COVID-19 is weak.

\subsection{Minerals}

Minerals are essential micronutrients that show a broad spectrum of functions including cellular metabolism, growth and thereby regulating overall health status (Chiu et al., 2021). Few minerals like zinc $(\mathrm{Zn})$, and selenium (Se), are reported to be effective against COVID-19, but other minerals like magnesium (Mg), calcium $(\mathrm{Ca})$, iron $(\mathrm{Fe})$, and copper $(\mathrm{Cu})$ also show mild to moderate effect against COVID-19.

\section{7. $\operatorname{Zinc}(Z n)$}

Zinc is one of the second most abundant minerals (trace element) used for growth, brain development, bone formation, and wound healing. The proposed mechanism of Zn against COVID-19, includes lowering the production, proliferation, and differentiation of various immune cells like NK cells, APCs, neutrophils and thus contributing to both innate and acquired immunity (Kumar et al., 2020). Zinc can also regulate the viral entry, fusion/attachment (endocytosis), and replication and translation (growth/proliferation) of various upper respiratory viruses (Ishida, 2019). Zn is reported to reduce the recombinant human ACE2 activity and may be helpful for treating COVID-19 (Speth et al., 2014). Studies show that chloroquine along with $\mathrm{Zn}$ supplementation may show a better anti-corona virus activity by inhibiting the attachment and subsequent multiplication of the SARS-CoV2 virus ( $\mathrm{Pal}$ et al., 2020; Ishida, 2019), however, this subject is somewhat controversial, especially in light of false claims related to hydroxychloroquine. In addition, $\mathrm{Zn}$ is reported to lower the production of pro-inflammatory cytokines via inhibiting the TLR and NFkB signaling pathways (Hamid and Thakur 2021). A clinical trial, conducted by Prasad et al. (2007) has shown that zinc supplementation in elderly subjects for one year could significantly decrease the incidence of viral infection through lowering of cytokines production and oxidative stress. Their data show that low levels of $\mathrm{Zn}$ ( $\mathrm{Zn}$ deficiency) are associated with high susceptibility of viral infection especially COVID-19. The author strongly suggested that all subjects (especially elderly people) maintain the normal $\mathrm{Zn}$ levels and in this way lower the risk of Covid infection and its severity.

\subsection{Selenium $(\mathrm{Se})$}

Selenium is an essential micronutrient used for various biological functions in the body, especially for immune regulation, anti-inflammation, and antioxidant properties. Se is usually incorporated as selenoprotein and used by cells for various biological functions especially for lowering oxidative stress (Chiu et al., 2021). The proposed mechanism of Se against COVID-19 includes possible enhancement of glutathione family antioxidants, and thus effectively lowering oxidative stress. Se can promote the production and proliferation of NK cells and thus play a major role in innate immunity (Avery and Hoffmann, 2018). Selenium administration can also downregulate various pro-inflammatory cytokines production (Mrityunjaya et al., 2020). Previous studies have indicated that Se supplementation could significantly lower the inflammatory response in respiratory distress syndrome patients (ARDS) as well as lowering the production of various pro-inflammatory cytokines (Mahmoodpoor et al., 2019). The above-mentioned antioxidant, anti-inflammatory, and immune-modulatory (innate immunity) activities of Se recommend itas a good choice for fighting against COVID-19 and its related complications.

Other minor minerals like iron $(\mathrm{Fe})$, and copper $(\mathrm{Cu})$ have also been found to show some positive impact against COVID-19 infection and severity. Iron supplementation showed lower inflammatory cytokines and altered immune cell activation as well as oxidative status (Wu et al., 2019). Similarly, copper intake was linked with antioxidant production and thus lowering oxidative stress in Covid infected subjects. In addition, $\mathrm{Cu}$ increased the production and proliferation of $\mathrm{T}$ and $\mathrm{B}$ cells, which contributed to antibodies production against the Coronavirus as well as killing several viruses like HIV, poliovirus and could inhibit the coronavirus infection (Raha et al., 2020).

\subsection{Combination therapy}

A few trials have been conducted by combining various dietary supplements (micronutrients) with functional foods/nutraceuticals due to their perceived holistic biological functions. However, the dosage, route of administration, duration, and combination ratio 


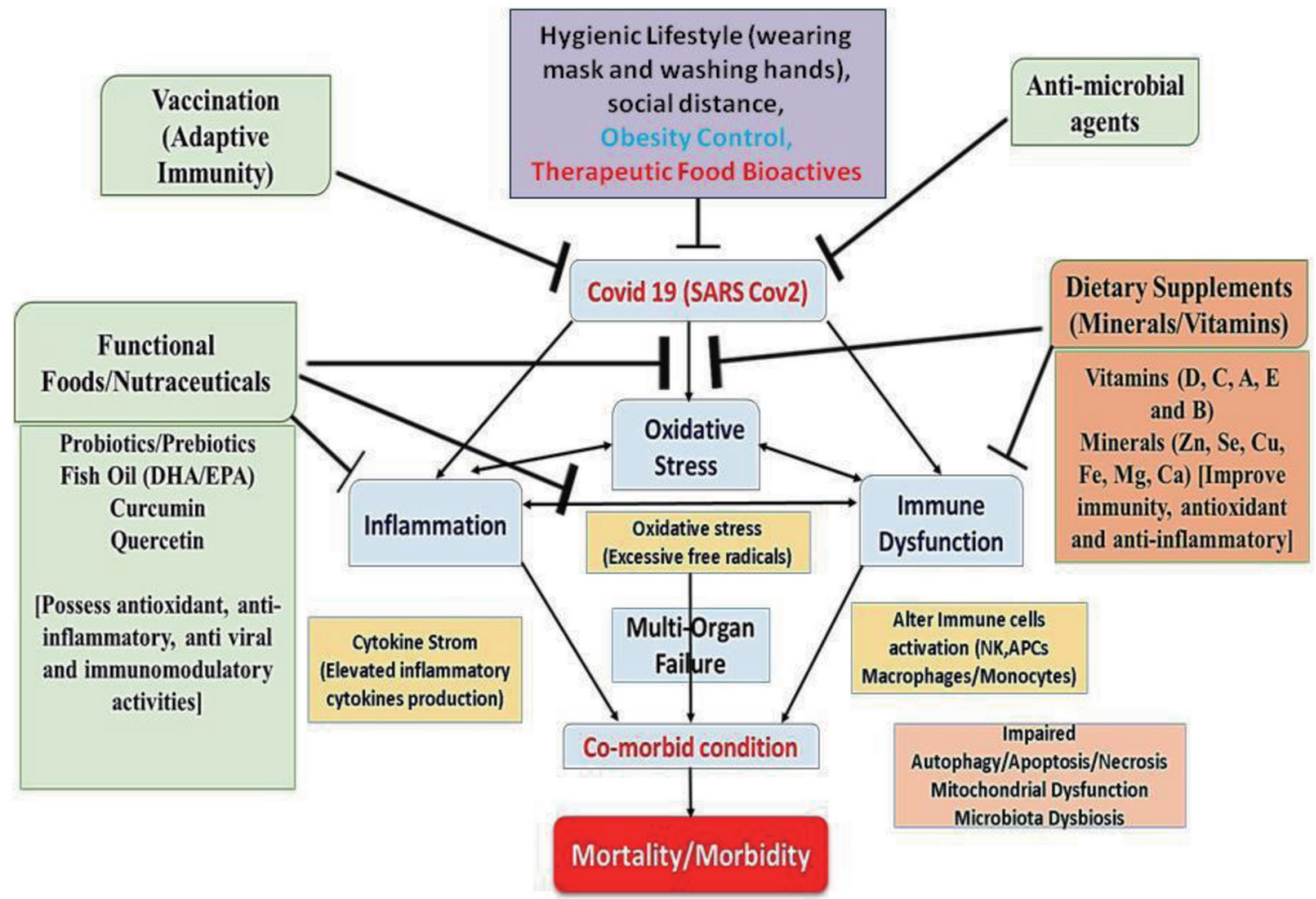

Figure 1. Overview of highlighting the importance of dietary supplements (micronutrients), functional foods/nutraceuticals in COVID-19.

remain to pose the biggest challenge (Colunga Biancatelli et al., 2020; Mittra et al., 2020). Likewise, dietary supplements (micronutrients), functional foods/nutraceuticals are combined with other standard anti-viral or anti-inflammatory drugs (synergistic effect). However again, the dose, duration, and combination ratio is an issue (Srivastava et al., 2021; Subedi et al, 2021). Figure 1 represents the overview of this review, highlighting the importance of dietary supplements (micronutrients), functional foods/nutraceuticals in improving health and immune system for use along with standard drugs and vaccines.

\section{Conclusion}

This coronavirus pandemic has challenged the entire health system globally and iys economy and has also killed several millions in the last few months. Here, we briefly provided the overall pathophysiology and its inter-connection and its subsequent consequences. The importance of hygienic lifestyle, body weight control and food habits to lower the severity of coronavirus infection were identified as important factors in relation to COVID-19. Finally, the importance of functional foods/nutraceuticals and dietary supplements (micronutrients) and how they may play a holistic function in improving overall health status in covid patients through suppressing oxidative stress, inflammation and positively regulating the immune response was reviewed. Currently limited number of clinical trials are available to support the importance of these various nutraceuticals/functional foods or dietary supplements against Coronavirus or Covid infection /Severity. Hence, further extensive clinical trials are needed to see if dietary supplements, functional foods/nutraceuticals along standard Covid vaccine (supportive therapy) would lower the ill effect of COVID-19 and its associated health issues. In addition, whether the dietary supplements, functional foods/nutraceuticals could lower the possible adverse effects of Covid vaccine deserves attention. Overall, this review provided some glimpse on the impact of various potential food bioactives against COVID-19 and their therapeutic effects and proposed possible mechanisms of action.

\section{References}

Abobaker, A., Alzwi, A., and Alraied, A.H.A. (2020). Overview of the possible role of vitamin C in management of COVID-19. Pharmacol. Rep. 72(6): 1517-1528.

Ahn, D.G., Shin, H.J., Kim, M.H., Lee, S., Kim, H.S., Myoung, J., Kim, B.T., and Kim, S.J. (2020). Current status of epidemiology, diagnosis, therapeutics, and vaccines for novel coronavirus disease 2019 (COVID-19). J. Microbiol. Biotechnol. 30(3): 313-324.

Akour, A. (2020). Probiotics and COVID-19: is there any link? Lett. Appl. Microbiol. 71(3): 229-234.

Alizon, S., Haim-Boukobza, S., Foulongne, V., Verdurme, L., TrombertPaolantoni, S., Lecorche, E., and Sofonea, M.T. (2021). Rapid spread of the SARS-CoV-2 Delta variant in some French regions, June 2021. Euro. Surveill. 26(28): 2100573.

Asher, A., Tintle, N.L., Myers, M., Lockshon, L., Bacareza, H., and Harris, W.S. (2021). Blood omega-3 fatty acids and death from COVID-19: A pilot study. Prostaglandins, Leukotrienes Essent. Fatty Acids 166: 102250.

Avery, J.C., and Hoffmann, P.R. (2018). Selenium, selenoproteins, and immunity. Nutrients 10(9): 1203.

Bakhshandeh, B., Sorboni, S.G., Javanmard, A.R., Mottaghi, S.S., Mehrabi, M.R., Sorouri, F., Abbasi, A., and Jahanafrooz, Z. (2021). Variants in ACE2; potential influences on virus infection and COVID-19 severity. 
Infect. Genet. Evol. 90: 104773.

Barlow, P.G., Svoboda, P., Mackellar, A., Nash, A.A., York, I.A., Pohl, J., and Donis, R.O. (2011). Antiviral activity and increased host defense against influenza infection elicited by the human cathelicidin LL-37. PLOS ONE 6(10): e25333.

Baud, D., Dimopoulou Agri, V., Gibson, G.R., Reid, G., and Giannoni, E. (2020). Using probiotics to flatten the curve of coronavirus disease COVID-2019 pandemic. Front. Public Health 8: 186.

Bhaskar, V., Yin, J., Mirza, A.M., Phan, D., Vanegas, S., Issafras, H., Michelson, K., Hunter, J.J., and Kantak, S.S. (2011). Monoclonal antibodies targeting IL-1 beta reduce biomarkers of atherosclerosis in vitro and inhibit atherosclerotic plaque formation in Apolipoprotein E-deficient mice. Atherosclerosis 216(2): 313-320.

Calder, P.C. (2007). Immunomodulation by omega-3 fatty acids. Prostaglandins, Leukotrienes Essent. Fatty Acids 77(5-6): 327-335.

Calder, P.C. (2021). Nutrition, immunity and COVID-19. Eur. J. Clin. Nutr. 75(9): 1309-1318.

Carr, A.C., and Rowe, S. (2020). The emerging role of vitamin C in the prevention and treatment of COVID-19. Nutrients 12(11): 3286.

Cascella, M., Rajnik, M., Aleem, A., Dulebohn, S., and Di Napoli, R. (2021). Features, evaluation, and treatment of coronavirus (COVID-19). StatPearls. StatPearls Publishing, Treasure Island (FL).

CDC, Food safety and coronavirus disease 2019 (COVID-19). (2019). Centers for Disease Control and Prevention website. https://www.cdc gov/foodsafety/newsletter/food-safetyand-Coronavirus.html. Updated May 11: 2020. Accessed May 19: 2020.

Chen, T.Y., Chen, D.Y., Wen, H.W., Ou, J.L., Chiou, S.S., Chen, J.M., and Hsu, W.L. (2013). Inhibition of enveloped viruses infectivity by curcumin. PLOS ONE 8(5): e62482.

Cheng, R.Z. (2020). Can early and high intravenous dose of vitamin C prevent and treat coronavirus disease 2019 (COVID-19)? Med. Drug Discovery 5: 100028.

Chiu, H.F., Venkatakrishnan, K., Golovinskaia, O., and Wang, C.K. (2021). Impact of Micronutrients on Hypertension: Evidence from Clinical Trials with a Special Focus on Meta-Analysis. Nutrients 13(2): 588.

Chong, H.X., Yusoff, N.A.A., Hor, Y.Y., Lew, L.C., Jaafar, M.H., Choi, S.B., and Liong, M.T. (2019). Lactobacillus plantarum DR7 improved upper respiratory tract infections via enhancing immune and inflammatory parameters: A randomized, double-blind, placebo-controlled study. J. Dairy Sci. 102(6): 4783-4797.

Colunga Biancatelli, R.M.L., Berrill, M., Catravas, J.D., and Marik, P.E. (2020). Quercetin and vitamin C: an experimental, synergistic therapy for the prevention and treatment of SARS-CoV-2 related disease (COVID-19). Front. Immunol. 11: 1451.

Colunga Biancatelli, R.M.L., Berrill, M., Catravas, J.D., and Marik, P.E. (2020). Quercetin and vitamin C: an experimental, synergistic therapy for the prevention and treatment of SARS-CoV-2 related disease (COVID-19). Front. Immunol. 11: 1451.

DAA. (2020). https://daa.asn.au/smart-eating-for-you/planning-your-pantry-during-the-Covid-19-pandemic

Daliu, P., Santini, A., and Novellino, E. (2019). From pharmaceuticals to nutraceuticals: Bridging disease prevention and management. Expert Rev. Clin. Pharmacol. 12(1): 1-7.

de Faria Coelho-Ravagnani, C., Campos Corgosinho, F., La Flor Ziegler Sanches, F., Marques Maia Prado, C., Laviano, A., and Felipe Mota, J. (2021). Dietary Recommendations During the COVID-19 Pandemic: an Extract. Kompass Nutrition \& Dietetics 1: 3-7.

Dehghani-Samani, A., Kamali, M., and Hoseinzadeh-Chahkandak, F. (2020). The Role of vitamins on the prevention and/or treatment of COVID-19 infection; A Systematic Review. Mod. Care J. 17(3): e104740.

FAO, Food and Agriculture Organization of the United Nations. (2020). Maintaining a healthy diet during the COVID-19 pandemic. http:// www.fao.org/3/ca8380en/CA8380EN.pdf. Published March 27, 2020. Accessed April 12, 2020.

Galanakis, C.M., Aldawoud, T., Rizou, M., Rowan, N.J., and Ibrahim, S.A. (2020). Food ingredients and active compounds against the coronavirus disease (COVID-19) pandemic: A comprehensive review. Foods 9(11): 1701.

Goossens, G.H., Dicker, D., Farpour-Lambert, N.J., Frühbeck, G., Mullerova, D., Woodward, E., and Holm, J.C. (2020). Obesity and COVID-19: A perspective from the European Association for the study of obesity on immunological perturbations, therapeutic challenges, and opportunities in obesity. Obes. Facts 13: 439-452.

Grant, W.B., Lahore, H., McDonnell, S.L., Baggerly, C.A., French, C.B., Aliano, J.L., and Bhattoa, H.P. (2020). Evidence that vitamin D supplementation could reduce risk of influenza and COVID-19 infections and deaths. Nutrients 12(4): 988.

Hagel, A.F., Layritz, C.M., Hagel, W.H., Hagel, H.J., Hagel, E., Dauth, W., and Raithel, M. (2013). Intravenous infusion of ascorbic acid decreases serum histamine concentrations in patients with allergic and nonallergic diseases. Naunyn-Schmiedeberg's Arch. Pharmacol. 386(9): 789-793.

Hamid, A.T., and Thakur, N.S. (2021). Role of functional food components in COVID-19 pandemic: A review. Ann. Phytomed. 10: S240-250.

Hussain, T., Tan, B., Yin, Y., Blachier, F., Tossou, M.C., and Rahu, N. (2016). Oxidative stress and inflammation: what polyphenols can do for us? Oxidative medicine and cellular longevity.

Iddir, M., Brito, A., Dingeo, G., Fernandez Del Campo, S.S., Samouda, H., La Frano, M.R., and Bohn, T. (2020). Strengthening the immune system and reducing inflammation and oxidative stress through diet and nutrition: considerations during the COVID-19 crisis. Nutrients 12(6): 1562.

Ishida, T. (2019). Review on the role of $\mathrm{Zn} 2+$ ions in viral pathogenesis and the effect of $\mathrm{Zn2}+$ ions for host cell-virus growth inhibition. Am. J. Biomed. Sci. Res. 2(1): 28-37.

Ismail, S.A., Saliba, V., Bernal, J.L., Ramsay, M.E., and Ladhani, S.N. (2021). SARS-CoV-2 infection and transmission in educational settings: a prospective, cross-sectional analysis of infection clusters and outbreaks in England. Lancet Infect. Disease 21(3): 344-353.

Jevalikar, G., Mithal, A., Singh, A., Sharma, R., Farooqui, K.J., Mahendru, S., and Budhiraja, S. (2021). Lack of association of baseline 25-hydroxyvitamin D levels with disease severity and mortality in Indian patients hospitalized for COVID-19. Sci. Rep. 11(1): 1-8.

Jovic, T.H., Ali, S.R., Ibrahim, N., Jessop, Z.M., Tarassoli, S.P., Dobbs, T.D., and Whitaker, I.S. (2020). Could vitamins help in the fight against COVID-19? Nutrients 12(9): 2550.

Junaid, K., Ejaz, H., Abdalla, A.E., Abosalif, K.O., Ullah, M.I., Yasmeen, H., and Rehman, A. (2020). Effective immune functions of micronutrients against SARS-CoV-2. Nutrients 12(10): 2992.

Kazemi, A., Mohammadi, V., Aghababaee, S.K., Golzarand, M., Clark, C.C., and Babajafari, S. (2021). Association of vitamin D status with SARSCoV-2 infection or COVID-19 severity: a systematic review and metaanalysis. Adv. Nutr. 12(5): 2040-2044.

Kellner, M., Noonepalle, S., Lu, Q., Srivastava, A., Zemskov, E., and Black, S.M. (2017). ROS signaling in the pathogenesis of Acute Lung Injury (ALI) and Acute Respiratory Distress Syndrome (ARDS). Adv. Exp. Med. Biol. 967: 105-137.

Kozlov, E.M., Ivanova, E., Grechko, A.V., Wu, W.K., Starodubova, A.V., and Orekhov, A.N. (2021). Involvement of oxidative stress and the innate immune system in SARS-CoV-2 infection. Diseases 9(1): 17.

Kumar, A., Kubota, Y., Chernov, M., and Kasuya, H. (2020). Potential role of zinc supplementation in prophylaxis and treatment of COVID-19. Med. Hypotheses. 144: 109848.

Laforge, M., Elbim, C., Frère, C., Hémadi, M., Massaad, C., Nuss, P., and Becker, C. (2020). Tissue damage from neutrophil-induced oxidative stress in COVID-19. Nat. Rev. Immunol. 20(9): 515-516.

Lee, G.Y., and Han, S.N. (2018). The role of vitamin E in immunity. Nutrients 10(11): 1614

Leisman, D.E., Ronner, L., Pinotti, R., Taylor, M.D., Sinha, P., Calfee, C.S., Hirayama, A.V., Mastroiani, F., Turtle, C.J., and Deutschman, C.S. (2020) Cytokine elevation in severe and critical COVID-19: a rapid systematic review, meta-analysis, and comparison with other inflammatory syndromes. Lancet Respir. Med. 12: 1233-1244.

Lewis, E.D., Meydani, S.N., and Wu, D. (2019). Regulatory role of vitamin E in the immune system and inflammation. IUBMB Life 71(4): 487-494.

Li, X., Geng, M., Peng, Y., Meng, L., and Lu, S. (2020). Molecular immune pathogenesis and diagnosis of COVID-19. J. Pharm. Anal. 10(2): 102108.

Liu, H., Chen, S., Liu, M., Nie, H., and Lu, H. (2020). Comorbid chronic diseases are strongly correlated with disease severity among COVID-19 
patients: a systematic review and meta-analysis. Aging Dis. 11(3) 668.

Liu, N., Sun, J., Wang, X., Zhang, T., Zhao, M., and Li, H. (2021). Low vitamin D status is associated with coronavirus disease 2019 outcomes: A systematic review and meta-analysis. Int. J. Infect. Dis. 104: 58-64.

Liu, Y., and Rocklöv, J. (2021). The reproductive number of the Delta variant of SARS-CoV-2 is far higher compared to the ancestral SARSCoV-2 virus. J. Travel. Med.

Lordan, R., Rando, H.M., and Greene, C.S. (2021). Dietary supplements and nutraceuticals under investigation for COVID-19 prevention and treatment. Msystems 6(3): e00122-21.

Mahmoodpoor, A., Hamishehkar, H., Shadvar, K., Ostadi, Z., Sanaie, S., Saghaleini, S.H., and Nader, N.D. (2019). The effect of intravenous selenium on oxidative stress in critically ill patients with acute respiratory distress syndrome. Immunol. Invest. 48(2): 147-159.

Martineau, A.R., Jolliffe, D.A., Hooper, R.L., Greenberg, L., Aloia, J.F., Bergman, P., and Camargo, C.A. (2017). Vitamin D supplementation to prevent acute respiratory tract infections: systematic review and meta-analysis of individual participant data. BMJ 356: i6583.

Mittra, I., de Souza, R., Bhadade, R., Madke, T., Shankpal, P.D., Joshi, M., and Badwe, R. (2020). Resveratrol and Copper for treatment of severe COVID-19: an observational study (RESCU 002). medRxiv.

Morrow, L.E., Kollef, M.H., and Casale, T.B. (2010). Probiotic prophylaxis of ventilator-associated pneumonia: a blinded, randomized, controlled trial. Am. J. Respir. Crit. Care Med. 182(8): 1058-1064.

Mortaz, E., Adcock, I.M., Folkerts, G., Barnes, P.J., Paul Vos, A., and Garssen, J. (2013). Probiotics in the management of lung diseases. Mediators Inflamm. 2013: 751068

Mrityunjaya, M., Pavithra, V., Neelam, R., Janhavi, P., Halami, P.M., and Ravindra, P.V. (2020). Immune-boosting, antioxidant and anti-inflammatory food supplements targeting pathogenesis of COVID-19. Front Immunol. 11: 570122

Murai, I.H., Fernandes, A.L., Sales, L.P., Pinto, A.J., Goessler, K.F., Duran, C.S., and Pereira, R.M. (2021). Effect of a single high dose of vitamin D3 on hospital length of stay in patients with moderate to severe COVID-19: a randomized clinical trial. JAMA 325(11): 1053-1060.

Pal, A., Squitti, R., Picozza, M., Pawar, A., Rongioletti, M., Dutta, A.K., and Prasad, R. (2021). Zinc and COVID-19: basis of current clinical trials. Biol. Trace. Elem. Res. 199: 2882-2892.

Pal, R., Banerjee, M., Bhadada, S.K., Shetty, A.J., Singh, B., and Vyas, A (2021). Vitamin D supplementation and clinical outcomes in COVID-19: a systematic review and meta-analysis. J. Endocrinol. Invest.

Pandey, K.R., Naik, S.R., and Vakil, B.V. (2015). Probiotics, prebiotics and synbiotics-a review. J. Food Sci. Technol 52(12): 7577-7587

Pereira, M., Dantas Damascena, A., Galvão Azevedo, L.M., de Almeida Oliveira, T., and da Mota Santana, J. (2020). Vitamin D deficiency aggravates COVID-19: systematic review and meta-analysis. Crit. Rev. Food Sci. Nutr.

Prasad, A.S., Beck, F.W., Bao, B., Fitzgerald, J.T., Snell, D.C., Steinberg, J.D., and Cardozo, L.J. (2007). Zinc supplementation decreases incidence of infections in the elderly: effect of zinc on generation of cytokines and oxidative stress. Am. J. Clin. Nutr. 85(3): 837-844.

Raha, S., Mallick, R., Basak, S., and Duttaroy, A.K. (2020). Is copper beneficial for COVID-19 patients? Med. Hypotheses. 142: 109814

Rocha, F.A.C., and de Assis, M.R. (2020). Curcumin as a potential treatment for COVID-19. Phytother. Res. 34(9): 2085-2087.

Rodriguez-Leyva, D., and Pierce, G.N. (2021). The Impact of Nutrition on the COVID-19 Pandemic and the Impact of the COVID-19 Pandemic on Nutrition. Nutrients 13(6): 1752.

Rogero, M.M., Leão, M.D.C., Santana, T.M., de MB Pimentel, M.V., Carlini, G.C., da Silveira, T.F., and Castro, I.A. (2020). Potential benefits and risks of omega- 3 fatty acids supplementation to patients with COVID-19. Free Radic. Biol. Med. 156: 190-199.

Shahidi, F. (2012). Nutraceuticals, functional foods and dietary supplements in health and disease. J. Food Drug. Anal. 20(1): 226-30.

Sharifi, A., Vahedi, H., Nedjat, S., Rafiei, H., and Hosseinzadeh-Attar, M.J. (2019). Effect of single-dose injection of vitamin D on immune cytokines in ulcerative colitis patients: a randomized placebo-controlled trial. Apmis 127(10): 681-687.

Sharma, S.V., Haidar, A., Noyola, J., Tien, J, Rushing, M., Naylor, B.M.,
Chuang, R.J., and Markham, C. (2020). Using a rapid assessment methodology to identify and address immediate needs among lowincome households with children during COVID-19. PLoS ONE 15(10): e0240009.

Shi, Y., Wang, Y., Shao, C., Huang, J., Gan, J., Huang, X., Bucci, E, Piacentini, M, Ippolito, G, and Melino, G (2020). COVID-19 infection: the per spectives on immune responses. Cell Death Differ. 27: 1451-1454.

Singh, P., Tripathi, M.K., Yasir, M., Khare, R., Tripathi, M.K., and Shrivastava, R. (2020). Potential inhibitors for SARS-CoV-2 and functional food components as nutritional supplement for COVID-19: a review. Plant Foods Hum. Nutr. 75: 458-66.

Smith, J.C., Sausville, E.L., Girish, V., Yuan, M.L., Vasudevan, A., John, K.M. and Sheltzer, J.M. (2020). Cigarette smoke exposure and inflammatory signaling increase the expression of the SARS-CoV-2 receptor ACE2 in the respiratory tract. Dev. Cell 53(5): 514-529.

Smith, M., and Smith, J.C. (2020). Repurposing therapeutics for COVID-19: Supercomputer-based docking to the SARS-CoV-2 viral spike protein and viral spike protein-human ACE2 interface. ChemRxiv.

Speth, R., Carrera, E., Jean-Baptiste, M., Joachim, A., and Linares, A (2014). Concentration-dependent effects of zinc on angiotensin-converting enzyme-2 activity (1067.4). FASEB J. 28: 1067-4.

Srivastava, S., Furlan, S.N., Jaeger-Ruckstuhl, C.A., Sarvothama, M., Berger C., Smythe, K.S., Garrison, S.M., Specht, J.M., Lee, S.M., Amezquita, R.A., Voillet, V., Muhunthan, V., Yechan-Gunja, S., Pillai, S.P.S., Rader, C., Houghton, A.M., Pierce, R.H., Gottardo, R., Maloney, D.G., and Riddell, S.R. (2021). Immunogenic Chemotherapy Enhances Recruitment of CAR-T Cells to Lung Tumors and Improves Antitumor Efficacy when Combined with Checkpoint Blockade. Cancer Cell 39(2): 193-208.e10.

Subedi, L., Tchen, S., Gaire, B.P., Hu, B., and Hu, K. (2021). Adjunctive Nutraceutical Therapies for COVID-19. Int. J. Mol. Sci. 22: 1963.

Sundararaman, A., Ray, M., Ravindra, P.V., and Halami, P.M. (2020). Role of probiotics to combat viral infections with emphasis on COVID-19. Appl. Microbiol. Biotechnol. 104(19): 8089-8104.

Syed, A. (2020). Coronavirus: a mini-review. Int. J. Curr. Res. Med. Sci 6(1): $8-10$

Szabo, Z., Marosvölgyi, T., Szabó, É., Bai, P., Figler, M., and Verzár, Z. (2020). The potential beneficial effect of EPA and DHA supplementation managing cytokine storm in coronavirus disease. Front. Physiol 11 752.

Tanumihardjo, S.A., Russell, R.M., Stephensen, C.B., Gannon, B.M., Craft, N.E., Haskell, M.J., and Raiten, D.J. (2016). Biomarkers of Nutrition for Development (BOND)-vitamin A review. J. Nutr. 146(9): 1816S1848 S.

UNICEF. (2020). Easy, affordable and healthy eating tips during the coronavirus disease (COVID-19) outbreak. United Nations Children's Fund (UNICEF) website. https://www.unicef.

Venkatakrishnan, K., Chiu, H.F., and Wang, C.K. (2019). Extensive review of popular functional foods and nutraceuticals against obesity and its related complications with a special focus on randomized clinical trials. Food Func. 10(5): 2313-2329.

Venkatakrishnan, K., Chiu, H.F., and Wang, C.K. (2019). Extensive review of popular functional foods and nutraceuticals against obesity and its related complications with a special focus on randomized clinical trials. Food Func. 10(5): 2313-2329.

Venkatakrishnan, K., Chiu, H.F., and Wang, C.K. (2019). Extensive review of popular functional foods and nutraceuticals against obesity and its related complications with a special focus on randomized clinical trials. Food Func. 10(5): 2313-2329.

Venkatakrishnan, K., Chiu, H.F., and Wang, C.K. (2019). Popular functional foods and herbs for the management of type-2-diabetes mellitus: a comprehensive review with special reference to clinical trials and its proposed mechanism. J. Funct. Food 57: 425-438.

Villamor, E., and Fawzi, W.W. (2005). Effects of Vitamin A Supplementation on Immune Responses and Correlation with Clinical Outcomes. Clin. Microbiol. Rev. 18(3): 446-464.

Wang, X., Hu, W., Zhu, L., and Yang, Q. (2017). Bacillus subtilis and surfactin inhibit the transmissible gastroenteritis virus from entering the intestinal epithelial cells. Biosci. Rep. 37(2): BSR20170082.

WHO. (2020). Nutrition advice for adults during the COVID-19 outbreak. 
World Health Organization Regional Office for the Eastern Mediterranean website. http://www.emro.who.int/nutrition/nutrition-infocus/nutrition-advice-for-adults-during-theCovid-19-outbreak.html. Published March 27, 2020. Accessed April 12, 2020.

Wiersinga, W.J., Rhodes, A., Cheng, A.C., Peacock, S.J., and Prescott, H.C. (2020). Pathophysiology, transmission, diagnosis, and treatment of coronavirus disease 2019 (COVID-19): a review. JAMA 324(8): 782 793.

Wu, D., Lewis, E.D., Pae, M., and Meydani, S.N. (2019). Nutritional modulation of immune function: analysis of evidence, mechanisms, and clinical relevance. Front. Immunol. 9: 3160.

Yuki, K., Fujiogi, M., and Koutsogiannaki, S. (2020). COVID-19 pathophysiology: A review. Clin. Immunol. 215: 108427.
Zahedipour, F., Hosseini, S.A., Sathyapalan, T., Majeed, M., Jamialahmadi, T., Al-Rasadi, K., and Sahebkar, A. (2020). Potential effects of curcumin in the treatment of COVID-19 infection. Phytother. Res. 34(11): 2911-2920.

Zeng, J., Wang, C.T., Zhang, F.S., Qi, F., Wang, S.F., Ma, S., and Wang, Y.P. (2016). Effect of probiotics on the incidence of ventilator-associated pneumonia in critically ill patients: a randomized controlled multicenter trial. Intensive Care. Med. 42(6): 1018-1028.

Zhang, J., Rao, X., Li, Y., Zhu, Y., Liu, F., Guo, G., and Peng, Z. (2021). Pilot trial of high-dose vitamin C in critically ill COVID-19 patients. Ann. Intensive Care 11(1): 1-12.

Zolkiewicz, J., Marzec, A., Ruszczyński, M., and Feleszko, W. (2020). Postbiotics-a step beyond pre-and probiotics. Nutrients 12(8): 2189. 